\title{
Optically Induced Reorientation in Nematic Cylindrical Structures ${ }^{\dagger}$
}

\author{
J. M. OTÓN, R. BELTRÁN and J. A. MARTÍN-PEREDA
}

Dept Electrónica Cuántica, ETSI Telecomunicación, 28040-Madrid, Spain

Cyclindrical structures of nematics give rise to several opto-optical effects related to molecular reorientation. One of these effects is the formation of diffraction ring patterns similar to the ones observed in planar cells, but differing in shape. Another effect has been observed, namely a quasi-chaotic motion of rings with a very large angular spread; this motion can be obtained using a $\mathrm{cw}$ laser and high power densities. The phenomenon could be attributed to thermal motion, however, there are some features that cannot be explained by a purely thermal effect, e.g., a wavelength dependence of the threshold and the frequencies of the ring motion.

\section{INTRODUCTION}

In the last few years, considerable attention has been paid to the optical-field-induced refractive index change in non-linear media such as nematic liquid crystals. ${ }^{1-3}$ Self-focusing, ${ }^{4}$ multiple-ring diffraction patterns, ${ }^{5}$ opto-optical modulation, ${ }^{6}$ four-wave mixing ${ }^{7}$ and other effects have been reported. Most of these effects can be achieved in lasers of moderate output power.

When a homeotropic orientation is induced in a cylindrical structure, ${ }^{8}$ this leads to a characteristic LC configuration known as "third dimension escape". In this configuration, LC molecules are perpendicularly oriented to the wall within its neighborhood, whereas LC molecules close to the symmetry axis of the structure are aligned parallel to it. The behavior of these kinds of structures in magnetic fields has been recently studied $;{ }^{9}$ it has been shown that there exists

$\uparrow$ Paper presented at the 10th International Liquid Crystal Conference, York, 15th21 st July 1984. 
a major dependence of the molecular reorientation on the relative orientation between the structure symmetry axis and the magnetic field.

The aim of this work is to study the behavior of homeotropic nematic LC cylindrical structures in a laser field. This cylindrical geometry is still seldomly used in optical studies of LC's. Greatest attention has been paid to planar structures, some work has also been done in planar-wedge structures, ${ }^{10,11}$ mainly devoted to electro- and magneto-optical effects. However, it was likely that the peculiar orientation of LC molecules in cylindrical homeotropic alignments should lead to new unexpected opto-optical phenomena. This paper summarizes our findings in this field.

\section{EXPERIMENTAL}

$\mathrm{N}$-(p-methoxybenzylidene)-p-n-butylaniline (MBBA) synthesized by $\mathrm{us}^{12}$ was selected as a nematic LC. The working temperature was $28-$ $30^{\circ} \mathrm{C}$. The devices used were glass microtubes, $0.2-0.8 \mathrm{~mm}$ inner diameter, prepared by cleaning with $4 \%$ hot DECON, rinsing with distilled water and absolute ethanol, and treating with boiling toluene saturated with hexadecyl-trimethyl ammonium bromide (HTAB). To avoid contamination, all the liquids were allowed to enter through the same tip of the tube, and extracted through the opposite end using a simple vacuum and trap system. Once the activation with HTAB was achieved, the inner wall was dried by an air or nitrogen stream using the same system. Then isotropic MBBA was allowed to fill up the tube, and the sample was finally sealed with parafilm at both ends. The LC orientation was checked by placing the sample between crossed polarizers in a microscope.

A $50 \mathrm{~mW}$ Spectra Physics He-Ne laser, as well as the $514.5 \mathrm{~nm}$ green and the $488.0 \mathrm{~nm}$ green-blue lines of a $20 \mathrm{~mW}$ tunable Spectra Physics $\mathrm{Ar}^{+}$ion laser, were used as input beams. Lenses with focal lengths from 2.5 to $10 \mathrm{~cm}$ were employed to focus the beam onto the sample, which was normal to the incident beam. The $\mathrm{Ar}^{+}$laser power could be varied from $0-20 \mathrm{~mW}$ in both lines. The He-Ne laser power was varied using a gradual reflectance beamsplitter. The power density of the focused beam may be up to $10 \mathrm{~kW} \mathrm{~cm}^{-2}$ in such conditions.

Patterns were projected onto a screen located $50 \mathrm{~cm}$ from the sample. A bright vertical line, owing to the lens effect of the horizontally placed sample, can be seen in all cases. The diffraction patterns appear on both sides of this line, distributed in a symmetrical fashion. 


\section{RESULTS}

\section{a) Ring patterns and opto-optical modulation}

As the input beam power is increasing, several effects can be observed. Firstly, a narrowing of the vertical line on the screen in seen, followed by the formation of an "onion-shell" diffraction pattern (Figure 1). This pattern is equivalent to the one found in planar samples, ${ }^{13}$ taking into account the deformation introduced by the cylindrical shape of the sample.

If the power is raised further, a new effect arises (Figure 2). Almost circular alternatively dark and bright rings appear, located outside the "onion-shell" pattern. These rings demonstrate a temporal random movement from the inner to the outer part of the image (Figures 2 and 3). A new set of random rings thicker than the first ones appear when the power is further raised (Figure 4). Finally, the maximum powers of either laser lead to scattering at these temperatures (28$30^{\circ} \mathrm{C}$ ), whereas at $22-25^{\circ} \mathrm{C}$ the last pattern (Figure 4) is maintained indefinitely; e.g., it has been kept for $15 \mathrm{~min}$. without changes. The same has been observed in the other patterns. There seems to be an energy transfer between the two outer sets of random rings, for they perform concerted movements, one of the sets becoming smaller as the other grows, and vice versa.

The above results have been obtained in samples whose symmetry axis was normal to the incident beam polarization. If the sample is placed parallel to the polarization, a similar set of patterns can be seen, but the power needed for each pattern to arise is smaller in the second case. The sample can be considered as a mixed homeotropichomogeneous structure, and it has been shown in planar structures of this kind the absence of a Freedericksz threshold. ${ }^{14}$

The random patterns show very large angular apertures, up to $60^{\circ}$ of half-angle; the "onion-shell" patterns give half-angles up to $10^{\circ}$.

If a light chopper is placed in the light path before the sample, and a power for static (onion-shell) patterns formation is selected, the light pulses create the corresponding pattern. If a second low power laser beam is allowed to pass through the same region, it will be modulated by the first laser, as happens in a planar sample. ${ }^{6}$ This kind of opto-optical modulation has been observed in our samples. However, we were unable to detect such a modulation in the random patterns, though the experimental set-up was unaltered.

\section{b) Thermal and wavelength dependences}

The power needed for the oscillating (random) pattern to appear 


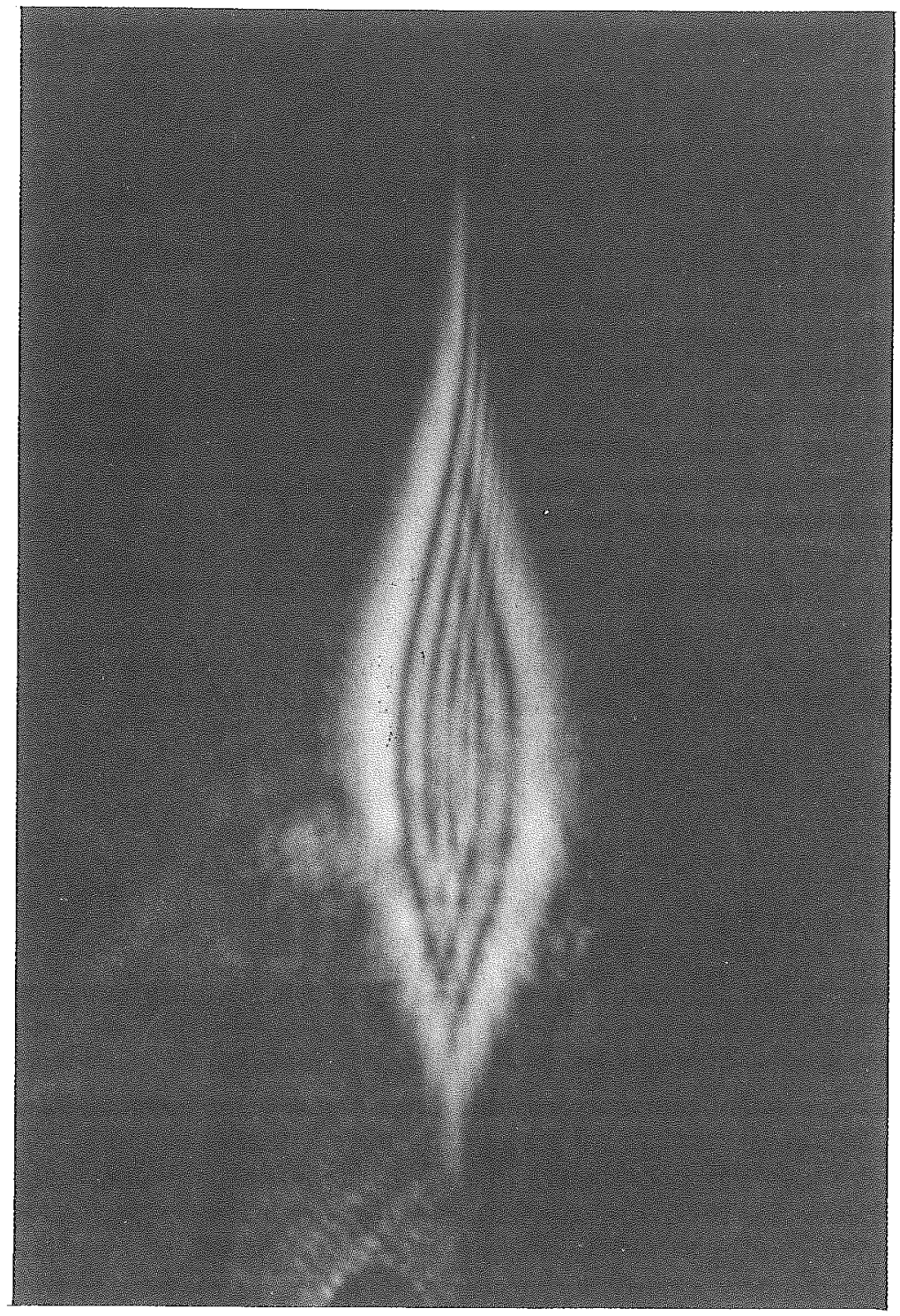

FIGURE 1 Static "onion shell" pattern obtained at low input powers. See Color Plate XVII, located in the final volume of these conference proceedings. 


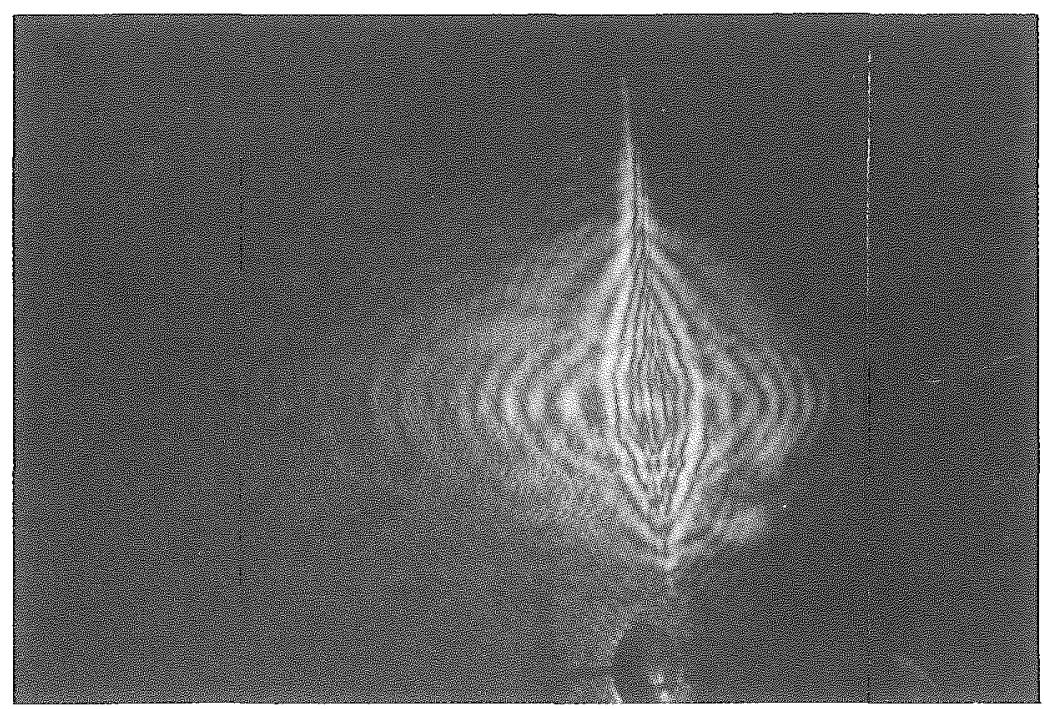

FIGURE 2 Start of the first ring motion set.

See Color Plate XVIII, located in the final volume of these conference proceedings.

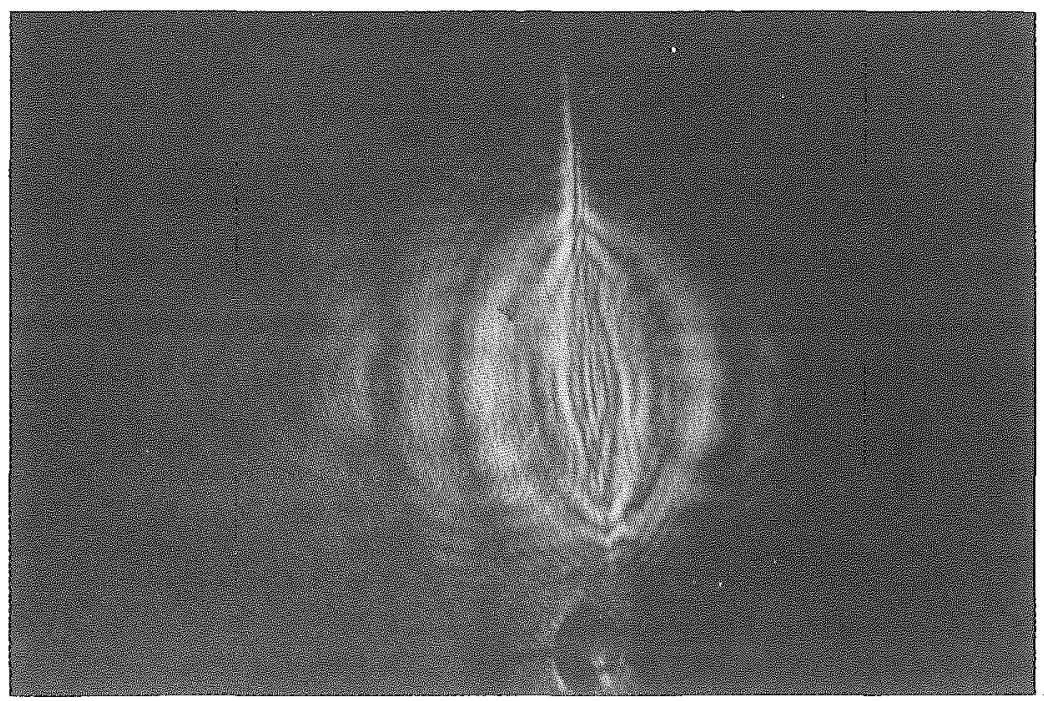

(a)

FIGURE 3 Evolution of the ring set of Figure 2.

See Color Plate XIX, located in the final volume of these conference proceedings. 


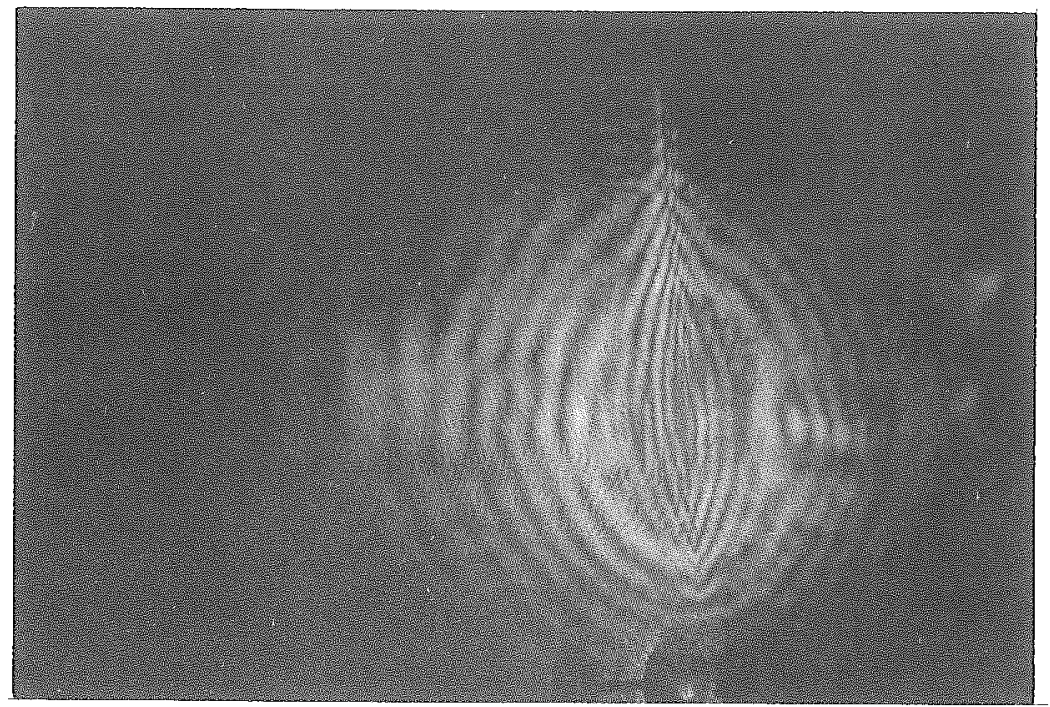

(b)

FIGURE 3b

See Color Plate XX, located in the final volume of these conference proceedings.

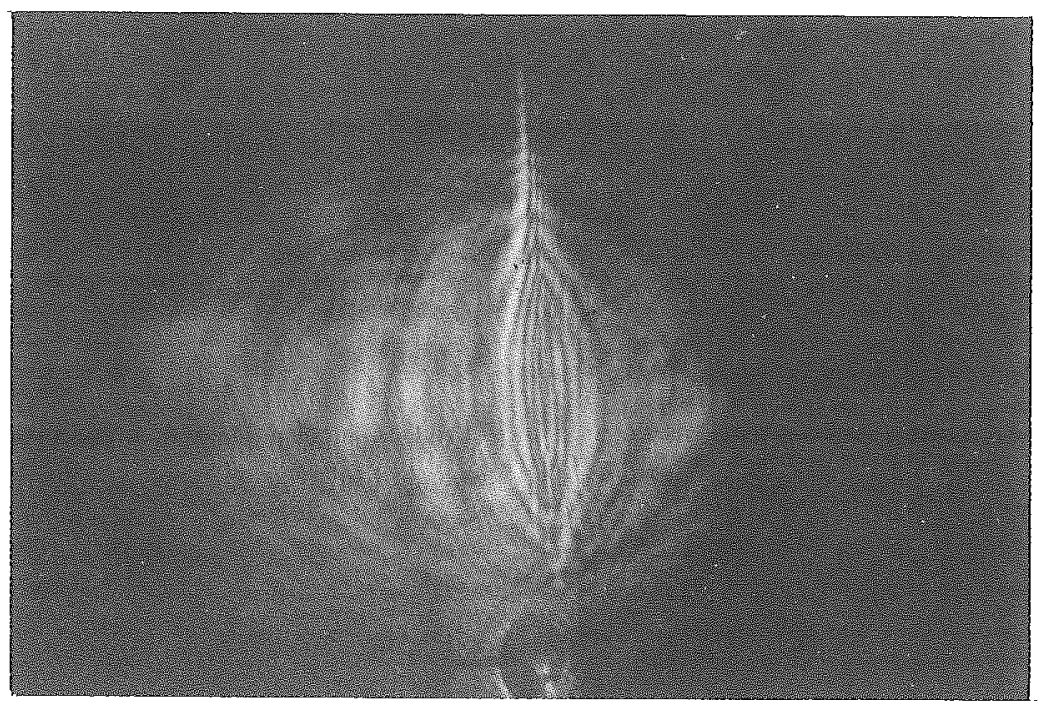

(c)

FIGURE $3 c$

See Color Plate XXI, located in the final volume of these conference proceedings. 


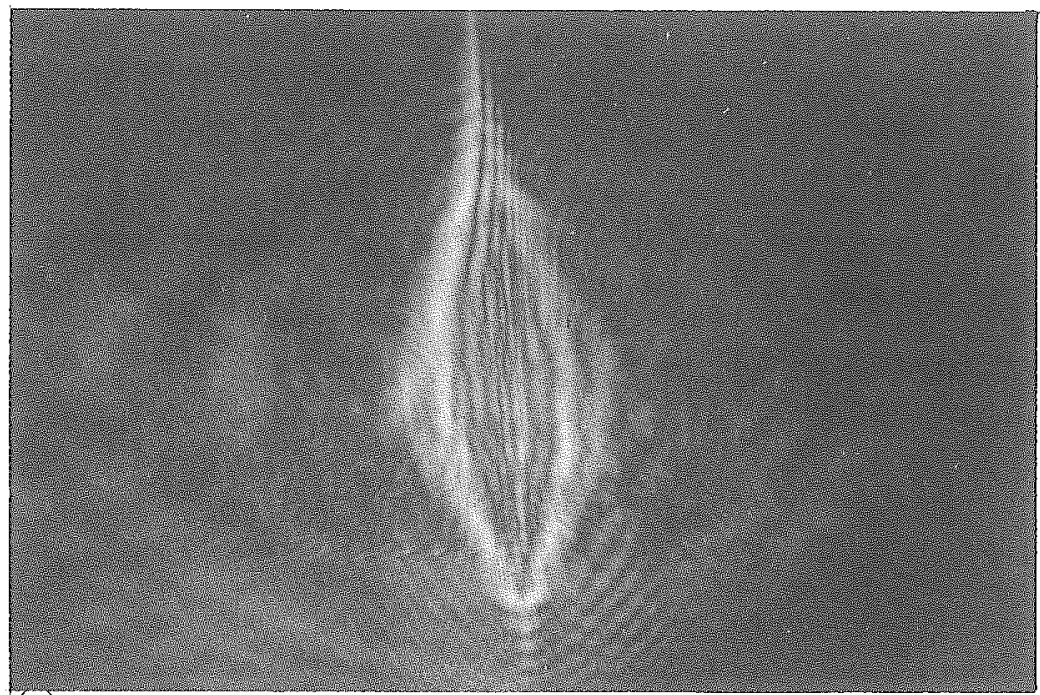

(a)

FIGURE 4 A second set of motion rings appears. The first set is still visible in the low part of the picture.

See Color Plate XXII, located in the final volume of these conference proceedings.

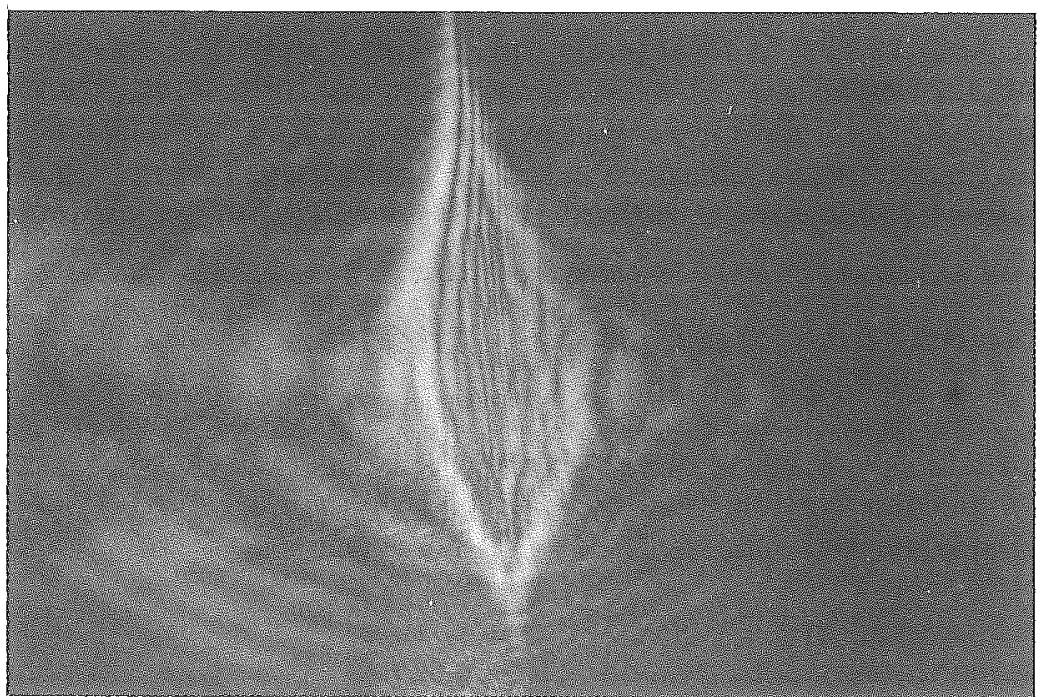

See Color Plate XXIII, located in the final volume of these conference proceedings. 


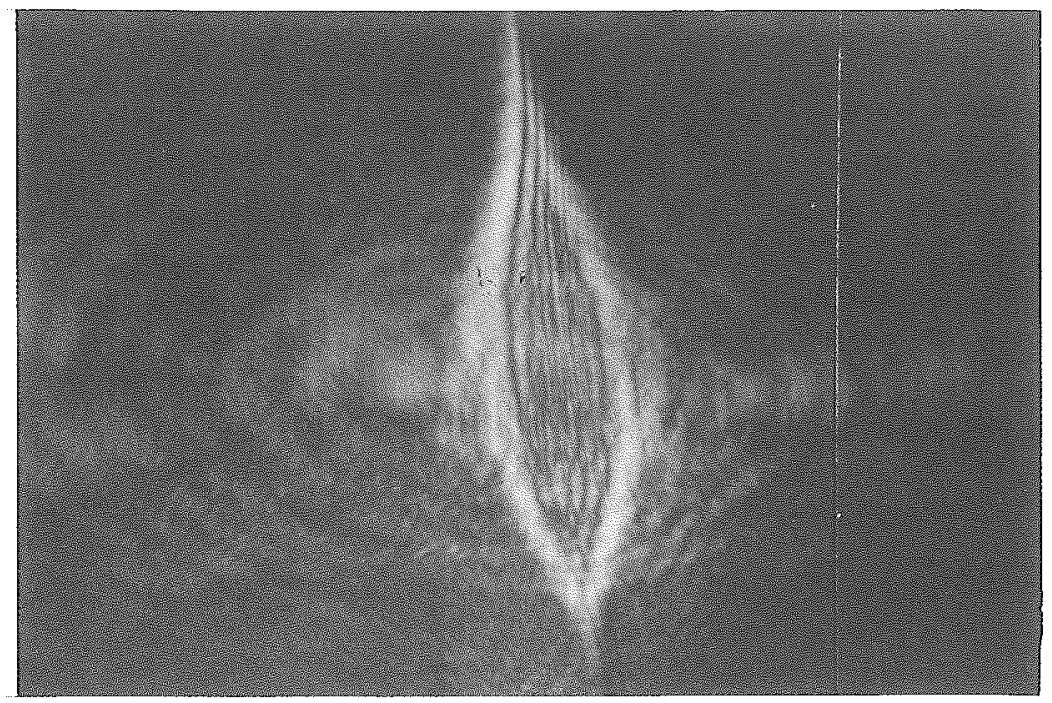

(c)

FIGURE 4c

See Color Plate XXIV, located in the final volume of these conference proceedings.

depends on several factors. Apart from a slight dependence on the sample diameter, a temperature dependence can be demonstrated: in fact, ring motions can be achieved with the following sequence: i) striking the sample with a power density slightly lower than its threshold for motion, and ii) warming up the sample with hot air. On the other hand, we have observed a marked dependence of this threshold on the exciting wavelength. Indeed, the power needed for the motion is much lower as the wavelength shortens, keeping constant the other experimental conditions. A $632.8 \mathrm{~nm} \mathrm{He}-\mathrm{Ne}$ laser beam requires three times as much power as the $488.0 \mathrm{~nm} \mathrm{Ar}^{+}$ion light to yield the oscillations; the power required in the $514.5 \mathrm{~nm}$ line of the same laser was about $25 \%$ more.

Similar sets of moving rings were detected in planar homeotropic structures. ${ }^{14}$ In those cases, the rings were almost perfectly circular in shape, and a concerted movement within the thicker and thinner ring sets was also found. The wavelength dependence was even more pronounced; i.e., we were unable to achieve the motion threshold with our $50 \mathrm{~mW}$ He-Ne laser, whereas $\mathrm{Ar}^{+} 514.5 \mathrm{~nm}$ green light leads to the motion only in warm samples $\left(30^{\circ} \mathrm{C}\right)$ at its maximum power $\left(20 \mathrm{~mW}\right.$; corresponding to $\left.2.2 \mathrm{~kW} \mathrm{~cm}^{-2}\right)$. The $488.0 \mathrm{~nm}$ line, however, needed only $12 \mathrm{~mW}\left(1.3 \mathrm{~kW} \mathrm{~cm}^{-2}\right)$ to produce the effect. 
Experimental conditions in planar samples were: $100 \mu \mathrm{m}$ thick homeotropic MBBA planar cells, 25 or $30^{\circ} \mathrm{C}$ and normal or slightly tilted incidence.

\section{c) Angular aperture}

Figure 5 is a typical plot of the aperture half-angle of our patterns vs. density power. These angles were measured on the screen horizontally, i.e. in the direction parallel to the sample rotation axis. After a small decrease at low powers, an increase corresponding to the static pattern can be seen (we have plotted the total half-cone angle and the outermost ring). The rise is abruptly interrupted by the appearence of the oscillating pattern; a further power increase does not modify the aperture of the static pattern.

The first part of the graph agrees with published results on planar samples, ${ }^{13}$ considering that our power densities depend on other experimental conditions, such as temperature, wavelength and sample thickness.

The inset of the same figure (Figure 5) represents the number of rings of the static pattern vs. power density. The data were taken by continuous sweeping of $\mathrm{Ar}^{+}$ion laser power, from powers equivalent to $0.5 \mathrm{~kW} \mathrm{~cm} \mathrm{~cm}^{-2}$ to $1.6 \mathrm{~kW} \mathrm{~cm} \mathrm{~cm}^{-2}$ and back again. Running time was $1 \mathrm{~min}$; some hysteresis can be observed in such a procedure.

\section{d) Frequency of the oscillating pattern}

A TIL 81 phototransistor working in photodiode mode was placed at several positions of the outer part (i.e., outside the static pattern) of the moving rings, and its output was recorded in a X-Y register. The purpose was to detect constant frequencies in the ring movement.

Figure 6 shows two examples of outputs at different sweep rates. Though frequency components between $2-20 \mathrm{~Hz}$ were detected, no pure frequencies were found.

\section{e) Behavior of other liquid crystals}

Several liquid crystals, other than MBBA, were tested under the same experimental conditions for the sake of comparing their behaviors:

1. Phase 5 (Merck)

2. Eutectic $(2: 1 \mathrm{w} / \mathrm{w})$ mixture of $\mathrm{p}$-cyanophenyl-p-heptyl benzoate and p-cyanophenyl-p-butylbenzoate (Eastman Kodak).

3. E7 (Merck) 


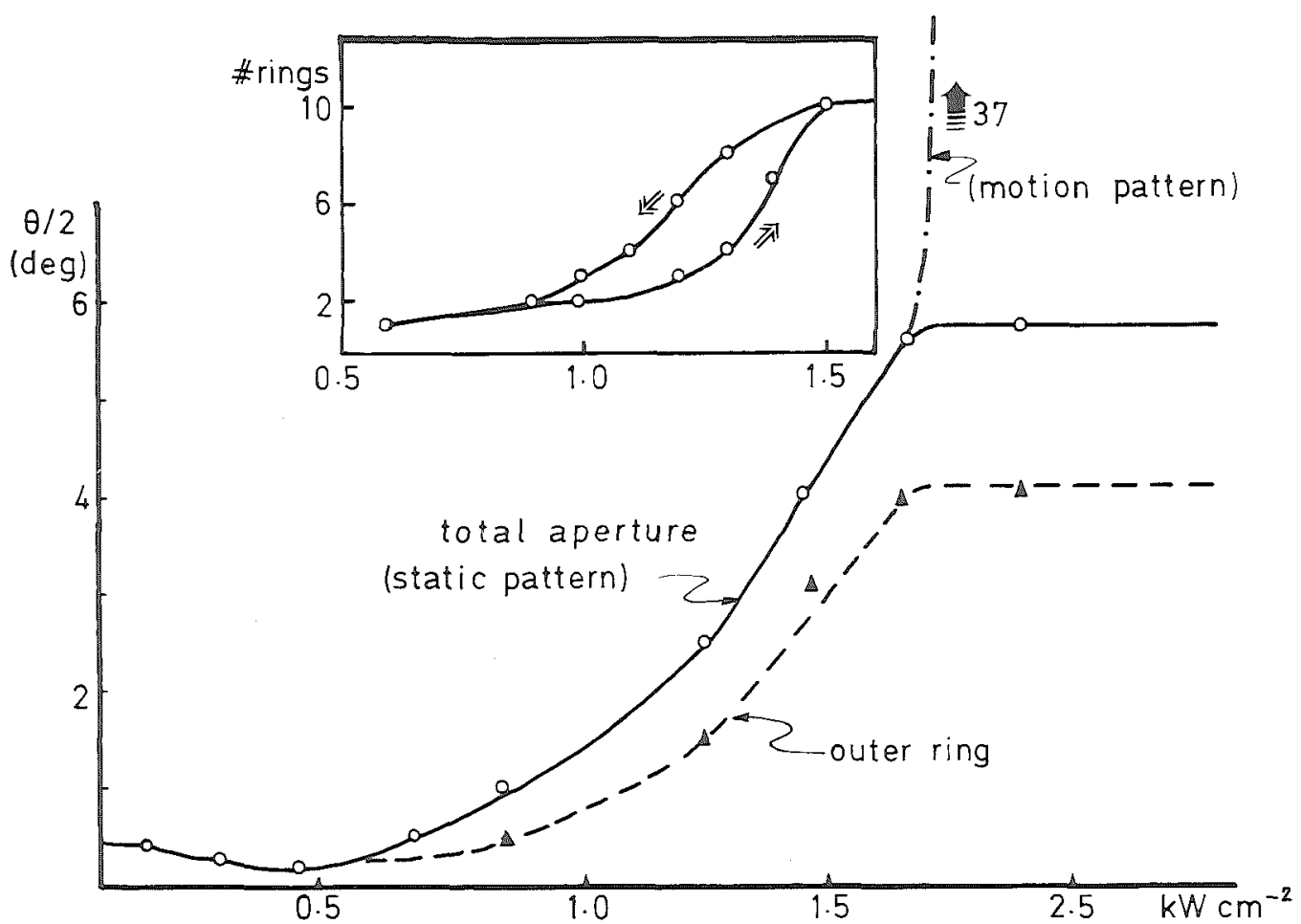

FIGURE 5 Half-angle aperture of the static pattern at different power densities. Inset: Number of rings of the same pattern in a 1 min. cycle from 0.5 to $1.6 \mathrm{~kW} \mathrm{~cm}{ }^{-2}$ and back. Experimental conditions: Sample diameter, $0.76 \mathrm{~mm}$; wavelength, $488.0 \mathrm{~nm}$; temperature, $25^{\circ} \mathrm{C}$. See text for details. 

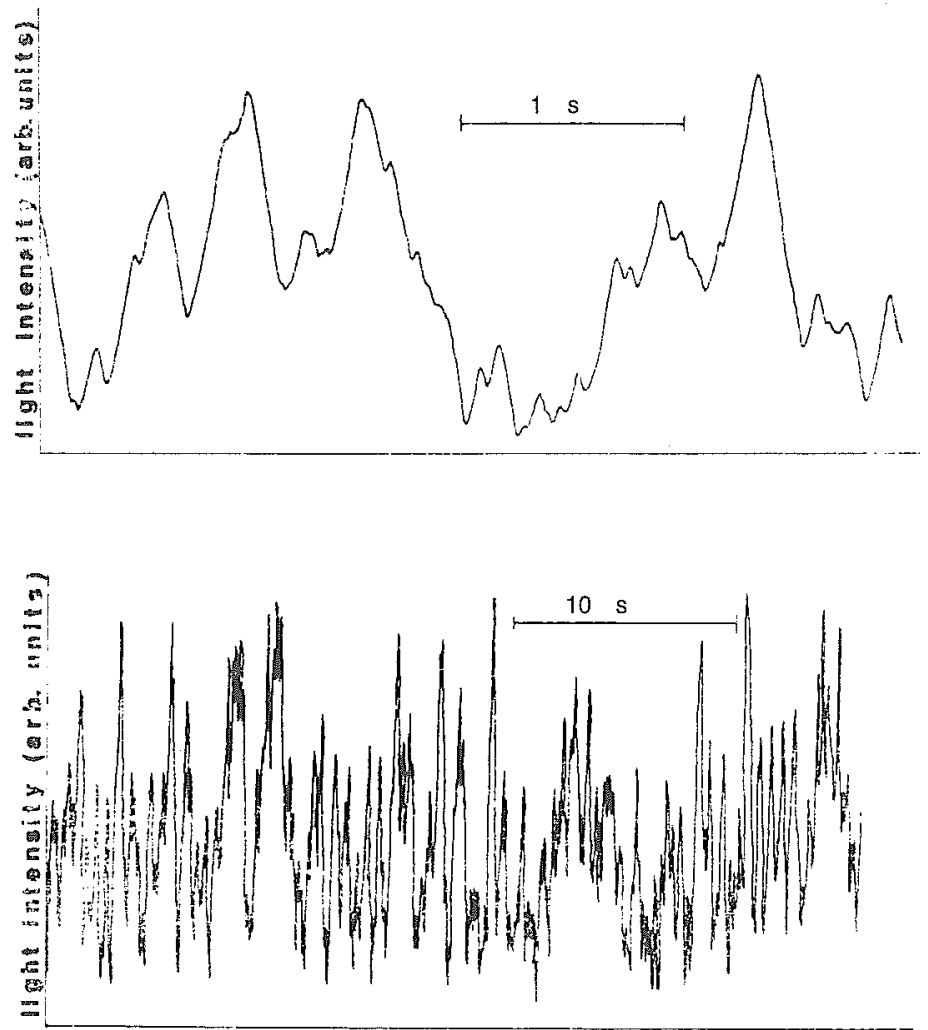

FIGURE 6 Photo-detector output of ring motion. The photo-detector was placed outside the static pattern, so only ring motion light is detected.

Phase 5 shows the same behavior as MBBA, but a previous warming up of the sample until about $5^{\circ} \mathrm{C}$ below the clearing point is required for the patterns to initiate. The oscillating pattern, on the other hand, is unstable in this case, i.e., it can be kept for no more than $5 \mathrm{~s}$. Nevertheless, this lack of stability is likely to be produced by the experimental arrangement itself, since the heating system, where a clear light path must be left, does not provide a confidently constant temperature in this range.

The eutectic mixture does not show any effect at all. In this case, the lack of response may be due to the low anisotropy exhibited by this mixture.

E7 shows the static pattern at the same temperatures as MBBA. However, oscillating patterns could not be induced, even at maximum laser powers and heating the sample until its clearing point. 


\section{DISCUSSION}

Two different kinds of rings are present in these experiments. The so-called static pattern can be ascribed to the ones previously reported ${ }^{13}$ in planar samples. The only difference, besides the shape of the sample, is that no threshold is expected in our case, due to the peculiar third-dimension-escape orientation of homeotropic cylindrical structures. Although the beam is focused onto the sample, the divergence of the beam is negligible throughout the sample diameter; therefore, a cylindrical shape of the beam can be considered.

In these conditions, the phase-shift calculation from Durbin et al. ${ }^{13}$ holds. This leads to an estimation of the output angle.

$$
\beta_{m} \simeq\left(\frac{d \Delta \Psi}{d \rho}\right)_{\max } /(2 \pi / \lambda)
$$

where

$$
\Delta \Psi(\rho)=\Delta \Psi_{0} \exp \left(-2 \rho^{2} / a^{2}\right)
$$

We consider a Gaussian profile of intensities, which produces a Gaussian profile of refractive indexes, owing to the different relative reorientation of the molecules. This assumption holds provided that the measurements are to be taken, in our case, in the direction parallel to the symmetry axis of the sample. Thus, the output wavefront will be phase-shifted in a Gaussian delay profile.

The maximum delay allowed in this model (i.e., the zero-point delay) comes from the difference between ordinary and extraordinary refractive indexes. On the other hand, the width of the Gaussian can be roughly estimated as the waist size. The outermost part of the diffraction pattern, as calculated from the inflection point slope, gives output half-angles of about $65^{\circ}$, thus covering all the angles found in other experiments.

However, though this model can satisfactorily account for the static pattern, it is unlikely that moving rings can be explained in such a way. There are two facts that point to an alternative explanation: the shape itself of the rings and the absence of opto-optical modulation in random patterns. Both facts can be explained assuming that the width of the sample region involved in the motion ring patterns is much smaller than the one forming the static pattern (about six times smaller, according to the half-angle ratio). Therefore, our diffraction patterns could be ascribed to a phase-shift profile composed by a 
centered inverted small gaussian on a six-times wider gaussian curve. Let us consider the origin of this "pit". The best candidate is a thermal effect. Indeed, there should be some kind of thermal dependence, as shown above. Moreover, it is known that the extraordinary refractive index decreases with temperature, in the nematic range of MBBA. Thus, a warming of the central region of the sample should lead to lower refractive indexes. On the other hand, there is a high absorption peak in the near UV spectrum of MBBA. The tail of this peak covers the blue-violet region of visible spectrum, being responsible for the yellow colour of this compound. Therefore, a light absorption is involved in the process, contributing to sample heating through relaxation.

The contribution of light absorption could explain why motion rings are detected in MBBA and Phase 5 (yellow) and not in E7 and the eutectic mixture (see Results). Also the wavelength dependence of the threshold for motion can be ascribed to absorption. Moveover, the presence of electronically excited states may alter the optical properties of the region, including its refractive index. The overall implications of this are still under study.

A temptative explanation of the oscillations themselves can be achieved in the same context. As the refractive index of the central region becomes lower (either by heating, absorption or both), the beam focus is displaced forward along beam axis. This leads to a lower light intensity on the warmed (and/or bleached) region, which eventually cools down (and/or colours) back again, and the process restarts.

\section{Acknowledgments}

The authors are indebted to Dr. M. A. Muriel for helpful discussions and the kind gift of an E7 sample. Financial support has been partially provided by the Spanish Comisión Asesora de Investigación Científica y Técnica.

\section{References}

1. R. M. Herman and R. J. Shenienko, Phys. Rev., A19, 1757 (1979).

2. I. C. Khoo, Phys. Rev., A23, 2077 (1981).

3. I. C. Khoo, Phys. Rev., A25, 10401636 (1982).

4. I. C. Khoo, S. L. Zhuang and S. Shepard, Appl. Phys. Lett, 39, 973 (1981).

5. S. D. Durbin, S. M. Arakelian and R. Shen, Phys. Rev. Lett., 47, 1411 (1981).

6. J. A. Martín-Pereda and F. J. López, Opt. Lett., 7, 590 (1982).

7. I. C. Khoo, Appl. Phys. Lett, 38 (3) 123 (1981).

8. P. G. deGennes, "The Physics of Liquid Crystals", Clarendon Press (Oxford, 1974). 
9. J. A. Martín-Pereda, M. A. Muriel and F. J. Sansa, Rev. Real Acad. Cienc. Exact. Fis, Nat., LXXV (4) 933 (1981).

10. J. A. Martín-Pereda and M. A. Muriel, J. Opt. Soc. Am., 70 (12) $1610(1980)$.

11. J. A. Martín-Pereda, M. A. Muriel and J. M. Otón, Appl. Opt., 23 (13) 2159 (1984).

12. P. Keller and L. Liebert, "Liquid Crystals for Physicists", in "Liquid Crystals", L. Liebert ed., Academic Press (New York, 1978).

13. S. D. Durbin, S. M. Arakelian and Y. R. Shen, Opt. Lett., 6 (9) 411 (1981).

14. F. J. López, J. A. Martín-Pereda and J. M. Otón, unpublished results. 